\title{
Das Folterverbot in der polizeilichen Praxis
}

Der »Fall Daschner« als Beleg für die rechtsstaatliche Absolutheit des Folterverbotes

Die Tätigkeit der Frankfurter Polizeibehörde im Rahmen der Ermittlungen wegen der Entführung des Jakob von Metzler steht seit nun knapp anderthalb Jahren im Fokus der Öffentlichkeit. Dies ist nicht zuletzt darauf zurückzuführen, daß die Staatsanwaltschaft beim Landgericht Frankfurt am Main ein Ermittlungsverfahren gegen den stellvertretenden Präsidenten der Frankfurter Polizeibehörde, Wolfgang Daschner, einleitete. Daschner wies einen Polizeibeamten an, Magnus G., der Jakob von Metzler entführt hatte, u.a. die Anwendung von Gewalt anzudrohen, um den Aufenthaltsort des Entführungsopfers zu erfahren. Außerdem hatte das Landgericht Frankfurt am Main darüber zu entscheiden, ob das Verhalten Daschners ein Verfahrenshindernis für den Strafprozeß gegen Magnus G. begründete (LG Frankfurt am Main, StV 2003, 327; dazu kritisch Weigend, StV 2003, 436). Wie aus zahlreichen Leserbriefen an diverse Tageszeitungen ersichtlich ist, überwog in der Öffentlichkeit die Einschätzung, Daschner habe nicht nur moralisch richtig, sondern auch in strafrechtlich nicht zu beanstandender Weise gehandelt. Demgegenüber bejahte die Staatsanwaltschaft Frankfurt am Main kürzlich einen hinreichenden Tatverdacht bezüglich einer Anstiftung zur Nötigung in einem besonders schweren Fall und erhob Anklage vor dem Landgericht Frankfurt am Main. Ob Daschner sich strafbar machte, ist Gegenstand dieses Beitrages. Zunächst wird der Sachverhalt dargestellt (A.), sodann die Entscheidung der Staatsanwaltschaft Frankfurt am Main wiedergegeben (B.) und schließlich die Strafbarkeit des Verhaltens von Daschner ausführlich beleuchtet (C.). In den Mittelpunkt der Betrachtung rückt die Frage nach der rechtlichen Zulässigkeit der staatlich veranlaßten Androhung von Gewalt zur Erlangung von Informationen. Dabei geht es inzident um polizei- und verfassungsrechtliche Grenzen polizeilichen Handelns, insbesondere die verschiedenen Ausprägungen des Folterverbotes.

\section{A. Sachverhalt}

Der stellvertretende Präsident der Frankfurter Polizeibehörde, Wolfgang Daschner, wies am Morgen des 01.10.2002 einen Polizeibeamten an, den der Entführung des elfjährigen Jakob von Metzler dringend verdächtigen Magnus G. unter Druck zu setzen. Daschner beauftragte einen Polizeibeamten, Magnus G. die Anwendung von Gewalt und den Einsatz von Drogen (»Wahrheitsserum «) anzudrohen, um den Aufenthaltsort des möglicherweise noch lebenden Entführten zu erfahren, nachdem Magnus G. schon

1 Der Sachverhalt beruht auf Angaben, die Artikeln in der Frankfurter Allgemeinen Zeitung $\mathrm{zu}$ entnehmen waren. Soweit der Wahrheitsgehalt einzelner Umstände, wie z.B. die von Magnus G. behauptete Androhung einer Vergewaltigung, streitig ist, bleiben diese außer Betracht. 
mehrfach falsche Aussagen über die ihm zur Last gelegten Taten gemacht hatte. Es stand zu befürchten, daß Jakob von Metzler schon mehrere Tage ohne Nahrung bzw. Obdach gefangen gehalten worden war. Der Festgenommene, so lautete die Anweisung, sei »nach vorheriger Androhung unter ärztlicher Aufsicht durch Zufügung von Schmerzen« zu befragen. Zum damaligen Zeitpunkt war noch nicht bekannt, daß Jakob von Metzler bereits tot war. Es war die Rede von »Schmerzen, die Sie nie in Ihrem Leben vergessen.« Nach der Drohung schilderte Magnus G. wahrheitsgemäß, er habe Jakob von Metzler erstickt und den Leichnam in einem Teich in Osthessen abgelegt.

Daschner fertigte einen Vermerk in der Vernehmungsakte an und informierte die Staatsanwaltschaft Frankfurt am Main über sein Vorgehen gegenüber dem Tatverdächtigen. Laut Aktenvermerk ging es Daschner »einzig und allein darum, das Kind so schnell wie möglich zu finden.« Er habe gehofft, daß Jakob von Metzler noch am Leben sei. Die Gewaltandrohungen hätten die Polizeibeamten zur Not auch umgesetzt. Dafür habe ein Kampfsportlehrer der Polizei bereit gestanden, der Magnus G. durch Überdehnen der Handgelenke Schmerzen habe zufügen sollen. Außerdem war daran gedacht, Druckstellen am Ohrläppchen zu erzeugen. Es sei ihm klar gewesen, daß sein Vorgehen juristisch überprüft werden würde. Für ihn, Daschner, sei es um eine Güterabwägung zwischen dem Recht des Kindes auf Leben und dem Recht des Angeschuldigten auf körperliche Unversehrtheit gegangen.

\section{B. Die Entscheidung der Staatsanwaltschaft Frankfurt am Main}

Die Staatsanwaltschaft Frankfurt am Main meint, Daschner habe sich nicht wegen einer Aussageerpressung hinreichend verdächtig gemacht. Vielmehr bestehe der hinreichende Tatverdacht, daß Daschner wegen einer Anstiftung zur Nötigung in einem besonders schweren Fall verurteilt werden könne. Die Staatsanwaltschaft Frankfurt am Main beantragte dementsprechend die Eröffnung des Hauptverfahrens. Wegen der besonderen Bedeutung des Falles erhob sie gem. § 24 I Nr. 3 GVG Anklage vor dem Landgericht Frankfurt am Main.

\section{Strafbarkeit des Verhaltens von Daschner}

Im folgenden wird die Strafbarkeit des Verhaltens von Daschner untersucht. In Betracht kommen Aussageerpressung (C.I.) sowie Anstiftung zur Nötigung (C.II.).

\section{Strafbarkeit wegen Aussageerpressung gem. $\$ \S 357$ I, 343 I Nr. 1 StGB}

Daschner machte sich nicht wegen einer Aussageerpressung gem. $\S \S 357$ I, 343 I Nr. 1 StGB strafbar. Es fehlt schon an der Erfüllung des objektiven Tatbestandes.

$\S 357$ I StGB setzt voraus, daß ein Vorgesetzter seinen Untergebenen zu einer rechtswidrigen Tat im Amt verleitet. Unter »rechtswidrige Tat im Amt« fallen alle Amtsdelikte. In Erwägung zu ziehen ist hier die rechtswidrige Verwirklichung des Tatbestandes der Aussageerpressung ( $\$ 343$ I Nr. 1 StGB) durch den ermittelnden Polizeibeamten. $\S 343$ I Nr. 1 StGB sieht vor, daß der Beamte zur Mitwirkung an 
einem Strafverfahren berufen war. Das heißt, daß der Amtsträger auf der Seite der Verfahrensführung dienstlich mitwirkte. ${ }^{2}$ Zum Strafverfahren gehören alle Verfahrensarten, welche die Möglichkeit zulassen, daß zumindest auch auf Strafe erkannt wird. Dazu zählen polizeiliche Ermittlungsverfahren. ${ }^{3}$ Den handelnden Polizeibeamten kam es aber darauf an, den Aufenthaltsort des entführten Jakob von Metzler zu erfahren. Es ging gerade nicht darum, ein Geständnis des dringend verdächtigen Magnus G. zu erhalten. Es handelte sich nicht um eine Maßnahme der Strafverfolgung (Repression), sondern um ein der Prävention zuzuordnendes Vorgehen. Ihr Verhalten diente aus Sicht der Polizeibeamten dem Schutz der körperlichen Integrität des vermeintlich noch lebenden Jakob von Metzler. Der bezweckte Nötigungserfolg muß sich im Rahmen des $\S 343$ StGB jedoch auf das Strafverfahren beziehen. ${ }^{4}$ Daher sind Ermittlungen zur Feststellung und Beseitigung eines polizeiwidrigen Zustandes nicht vom Tatbestand des $\S 343$ StGB erfaßt. ${ }^{5}$ Demzufolge ist $\S 343$ I Nr. 1 StGB tatbestandlich nicht erfüllt. ${ }^{6}$ Daher liegt auch keine »rechtswidrige Tat im Amt « im Sinne von $§ 357$ I StGB vor.

\section{Strafbarkeit wegen Anstiftung zur Nötigung gem. $§ \$ 240$ I, 26 StGB}

\section{Weisung des Daschner als Bestimmen zu einer Nötigung}

Daschner bestimmte einen ermittelnden Polizeibeamten zu einer vorsätzlichen, rechtswidrigen Haupttat im Sinne von $§ 26$ StGB. Er wies diesen an, Magnus G. die Anwendung von Gewalt sowie den Einsatz von Drogen anzukündigen. Der dieser Weisung nachkommende Polizeibeamte nötigte Magnus G., indem er ihm drohte. Unter Drohung im Sinne von $\S 240$ I StGB versteht man das Inaussichtstellen eines empfindlichen Übels, auf dessen Eintritt der Täter Einfluß hat oder zu haben vorgibt. ${ }^{7}$ Der Polizeibeamte teilte Magnus G. mit, man werde ihm - wenn er nicht den Aufenthaltsort des Jakob von Metzler nenne - Schmerzen zufügen, die er »sein Leben lang « nicht vergessen werde. Es war beabsichtigt, die Handgelenke des Magnus G. zu überdehnen und Druckstellen am Ohrläppchen zu erzeugen.

Dadurch erreichte der vorsätzlich handelnde Polizeibeamte, daß Magnus G. offenbarte, er habe Jakob von Metzler erstickt und den Leichnam in einem Teich in Osthessen versenkt.

2 Systematischer Kommentar-Horn/Wolters, StGB, 7. Auflage 2002, § 343, Rn. 3 a.

3 Systematischer Kommentar-Horn/Wolters, aaO (Fn. 2), § 343, Rn. 4.

4 Systematischer Kommentar-Horn/Wolters, aaO (Fn. 2), § 343, Rn. 3 a; Haurand/Vahle, NVwZ 2003, 513, 519.

5 BGHSt 6, 144; Leipziger Kommentar-Jescheck, StGB, 10. Auflage 1988, § 343, Rn. 4; Tröndle/Fischer, StGB, 51. Auflage 2003, § 343, Rn. 5. Ebenso Miehe, NJW 2003, 1219, 1220 , in bezug auf die physische Erzwingung einer Auskunft zur Gefahrenabwehr.

6 Grasnick, FAZ Nr. 97 vom 26.04.2003, S. 43, erste, zweite und sechste Spalte; Haurand/ Vahle, aaO (Fn. 4), 519; Schreiber, NJW 2003, Heft 23, XII, XIV; so auch i.E. Lackner/ Kühl, StGB, 24. Auflage 2001, § 343, Rn. 4, der bei einem Handeln aussschließlich zum Zweck der Prävention den subjektiven Tatbestand verneint. Wohl a.A. Schäfer, NJW 2003, 947 und Jerouschek/Kölbel, JZ 2003, 613, 619.

7 Tröndle/Fischer, StGB, aaO (Fn. 5), § 240, Rn. 31. 
Im Rahmen von $\S 240$ I StGB wird die Rechtswidrigkeit des Handelns nicht durch das tatbestandsmäßige Verhalten des Täters indiziert. Es bedarf zum einen der ausdrücklichen Feststellung, daß keine Rechtfertigungsgründe gegeben sind, zum anderen muß die Tat verwerflich sein. Der die Weisung ausführende Polizeibeamte war nicht gerechtfertigt. Ein strafrechtlicher Rechtfertigungsgrund ergibt sich weder aus dem Hessischen Gesetz über die öffentliche Sicherheit und Ordnung (HSOG) ${ }^{8}$ noch aus dem Strafgesetzbuch. ${ }^{9}$ Zudem ist die Verwerflichkeit der Tat im Sinne von § 240 II StGB zu bejahen. ${ }^{10}$

\section{a) Rechtfertigung im Polizeirecht?}

Die tatbestandliche Handlung eines Trägers hoheitlicher Gewalt kann aufgrund einer entsprechenden gesetzlichen Ermächtigungsgrundlage gerechtfertigt sein, soweit deren Voraussetzungen vorliegen. ${ }^{11}$ Der ermittelnde Polizeibeamte konnte sich jedoch nicht auf $\S 12$ I 1 HSOG i.V.m. $\S \$ 47$ ff. HSOG stützen.

§ 12 I 1 HSOG läßt eine Befragung zu, wenn tatsächliche Anhaltspunkte dafür bestehen, daß die zu befragende Person sachdienliche Angaben zur Aufklärung des Sachverhalts in einer bestimmten polizeilichen Angelegenheit machen kann. Befragen ist jedes Verhalten, das auf die Erlangung von Informationen durch Auskünfte seitens der befragten Person in einer polizeilichen Angelegenheit zielt. ${ }^{12}$ An dieser Stelle muß eine Abgrenzung zu strafrechtlichen Ermittlungen erfolgen. Diese stellen gerade keine polizeiliche Angelegenheit dar. ${ }^{13}$ Magnus G. sollte dazu bewegt werden, den Aufenthaltsort des entführten Jakob von Metzler preiszugeben, um sodann Gefahren für Leib und Leben des Entführungsopfers abwenden zu können. Damit stellt sich der aufzuklärende Sachverhalt als Gefahr dar, die ohne die Information der befragten Person nicht abgewehrt werden kann. Es ging um Prävention, nicht um Repression. ${ }^{14}$ Magnus G. war als Störer gem. $§ 12$ II 1 HSOG auskunftspflichtig. ${ }^{15}$

Allerdings besteht ein Aussageverweigerungsrecht, wenn die Auskunft die Gefahr für den Befragten erhöht, wegen einer Straftat verfolgt zu werden. Das folgt aus dem rechtsstaatlichen Grundsatz, daß niemand gezwungen ist, sich selbst zu belasten (»nemo tenetur se ipsum accusare «). ${ }^{16}$ Dieses Prinzip findet seinen Niederschlag in $\S 12$ II 2 HSOG. Danach hat die betroffene Person unter den Voraussetzungen von $\S \S 52$ bis 55 StPO ein Auskunftsverweigerungsrecht. Jedoch gilt dieses nicht für den Fall, daß die Auskunft für die Abwehr für Leib, Leben oder Freiheit einer Person erforderlich ist ( $\$ 12$ II 3 HSOG). Magnus G. durfte deshalb nicht die Auskunft verweigern, er war gem. $\S 12$ II 1 HSOG auskunftspflichtig. Damit ist aber noch nicht

8 C.II.1.a).

9 C.II.1.b).

10 C.II.1.d).

11 Leipziger Kommentar-Hirsch, StGB, 11. Auflage 1994, Vor § 32, Rn. 142 ff.

12 Rachor, F Rn. 258, in: Lisken/Denninger, Handbuch des Polizeirechts, 3. Auflage 2001.

13 Rachor, aaO (Fn. 12), F Rn. 269.

14 Siehe bereits unter C.I.

15 Zur Auskunftspflicht umfassend Rachor, aaO (Fn. 12), F Rn. 277 ff.

16 Haurand/Vahle, aaO (Fn. 4), 517. 
gesagt, daß die Androhung von Gewalt zur Durchsetzung der Auskunftspflicht zulässig war. Gegen deren Rechtmäßigkeit spricht, daß nach § 12 IV HSOG die Regelung des $\S 136$ a StPO entsprechende Anwendung findet. ${ }^{17}$ Nach $\S 136$ a I 1 StPO ist die Freiheit der Willensentschließung und -betätigung als Ausfluß von Art. 1 I 1 GG $^{18}$ umfassend vor Beeinträchtigungen im Rahmen der Vernehmung geschützt. Als verbotene Vernehmungsmethoden werden in $\S 136$ a I 1 StPO Mißhandlungen sowie körperliche Eingriffe aufgeführt. Mißhandlung ist jede erhebliche Beeinträchtigung der körperlichen Unversehrtheit oder des körperlichen Wohlbefindens. ${ }^{19}$ Körperliche Eingriffe sind Maßnahmen, die sich unmittelbar auf den Körper des Beschuldigten auswirken. ${ }^{20}$ Aber nicht nur die Anwendung derartiger Methoden ist unzulässig. Bereits die Drohung, sich solcher Zwangsmaßnahmen zu bedienen, ist verboten, § 136 a I 3 StPO. Das gleiche gilt nach der ausdrücklichen Verweisung in § 12 IV HSOG auch für die Präventivzwecken dienende Befragung im Sinne von § 12 I HSOG. Ebenfalls unzulässig ist die Anwendung unmittelbaren Zwanges »zur Abgabe einer Erklärung « (§ 52 II HSOG). Darunter fällt auch die Androhung derartiger Zwangsmittel. ${ }^{21}$

Dagegen spricht nicht, daß es sich bei der hier erstrebten Information über den Aufenthaltsort des Entführungsopfers nicht um eine rechtlich bindende Äußerung handelt. Zwar läßt es der Wortlaut von § 52 II HSOG zu, unter »Erklärung « nur eine Äußerung zu verstehen, welche in bindender Form abgegeben wird. Eine derartige Auslegung der Norm hätte aber zur Folge, daß ihr Anwendungsbereich äußerst schmal würde. Es läßt sich kaum ein Fall denken, in welchem unmittelbarer Zwang eingesetzt werden könnte, um die Abgabe einer rechtlich bindenden Erklärung zu erreichen. ${ }^{22}$ Folglich können unter »Erklärung « im Sinne von § 52 II HSOG jegliche Äußerungen subsumiert werden, ohne daß es auf einen Rechtsbindungswillen ankommt. ${ }^{23}$ Aus den polizeirechtlichen Normen ist zwingend zu schließen, daß die Androhung von Gewalt im Rahmen der Befragung rechtswidrig war.

Allerdings könnte man anführen, daß der Ausschluß von Zwang bei Befragungen bzw. Vernehmungen auf der »generellen Hilflosigkeit des Festgenommenen« gegenüber der Polizei gründe. Hier sei die Ausgangssituation genau umgekehrt, da der Entführer die Situation in der Hand habe. Nur er wisse, wo das Opfer versteckt ist. Diese Konstellation spiegele sich in § 12 IV HSOG nicht wider. Es liege eine »Bewertungslücke« vor. ${ }^{24}$ Unter »Bewertungslücke« lässt sich demnach eine Konstellation verstehen, in der die Rechtsordnung eine bestimmte Norm bereitstelle und insofern eine

17 Hecker, KJ 2003, 210, 212. Insofern ist es verkürzt, wenn Hamm, NJW 2003, 946, von einem unmittelbaren Verstoß gegen $\S 136$ a StPO ausgeht.

18 Meyer-Goßner, StPO, 46. Auflage 2003, § 136 a, Rn. 1.

19 Meyer-Goßner, aaO (Fn. 18), § 136 a, Rn. 7.

20 Meyer-Goßner, $\mathrm{aaO}$ (Fn. 18), § 136 a, Rn. 9.

21 Baurand/Vahle, aaO (Fn. 4), 518; Schreiber, aaO (Fn. 6), XIV.

22 Beispielsweise wird die theoretisch denkbare Konstellation, Gefahrenabwehr- und Polizeibehörden könnten den Bürger mittels unmittelbaren Zwanges zur Abgabe einer Erklärung bewegen, die auf den Schluß eines öffentlich-rechtlichen Vertrages gerichtet ist, praktisch wohl kaum Bedeutung erlangen.

23 So auch Rachor, aaO (Fn. 12), F Rn. 259 und Rn. 782.

24 Brugger, FAZ Nr. 58 vom 10.03.2003, S. 8, dritte Spalte. 
rechtliche Bewertung vornehme, welche einer anderen Norm der Rechtsordnung widerspreche. ${ }^{25}$ Diese »Bewertungslücke« sei durch eine teleologische Reduktion des Anwendungsbereichs der Norm juristisch zu bewältigen. ${ }^{26}$

Allerdings ist dies schon methodisch nicht haltbar. So fehlt es bereits an einer Lücke, wenn man die einschlägigen Vorschriften des Grundgesetzes, der Konvention zum Schutze der Menschenrechte und Grundfreiheiten (Europäische Menschenrechtskonvention $=\mathrm{EMRK})^{27}$ sowie des UN-Übereinkommens gegen Folter und andere grausame, unmenschliche oder erniedrigende Behandlung oder Strafen (Anti-Folterkonvention) ${ }^{28}$ berücksichtigt. Diese haben Eingang in die Wertung des § 12 IV HSOG gefunden.

Ausgangspunkt der Betrachtung ist Art. 1 I GG. Die Würde jedes Menschen, auch diejenige eines Störers oder Straftäters, ist schrankenlos geschützt. Bei der Menschenwürde handelt es sich nicht um einen absoluten Begriff. ${ }^{29}$ Vielmehr kann »nur in Ansehung des konkreten Falles « entschieden werden, ob die Menschenwürde verletzt ist. ${ }^{30}$ Ansatzpunkt ist also die Beurteilung eines Vorgangs als Verletzung. ${ }^{31}$

Die Menschenwürde von Magnus G. wäre nicht tangiert, stellte man lediglich darauf $\mathrm{ab}$, daß ihm angedroht wurde, seine Handgelenke zu überdehnen. Dies gilt selbst dann, wenn der Polizeibeamte es nicht bei der Androhung belassen, sondern zur Tat geschritten wäre. Anderenfalls würde jede staatliche Zwangsmaßnahme (zum Beispiel das Arretieren mittels Handschellen) ohne weiteres in die Menschenwürde des Betroffenen eingreifen und - weil die Menschenwürde gem. Art. 1 I GG unantastbar ist verfassungswidrig sein. Art. 1 I GG darf aber gerade vor dem Hintergrund seiner Genese nicht zur »kleinen Münze« verkommen. ${ }^{32}$ Daher können die erwähnten Handlungen für sich genommen in die Menschenwürde nicht eingreifen. Eingriffe in das Leben oder die körperliche Unversehrtheit sind vielmehr grundsätzlich allein an Art. 2 II GG zu messen. ${ }^{33} \mathrm{Zu}$ berücksichtigen sind aber die Begleitumstände der Eingriffshandlung, namentlich die objektive Zielrichtung des staatlichen Handelns. ${ }^{34}$ Hier war die Androhung von Gewalt kein Selbstzweck, sondern sie erfolgte, um Magnus G. zur Abgabe einer Äußerung zu bewegen. Dieser Umstand darf bei der juristischen Betrachtung nicht ausgeblendet werden. Dadurch wird der Betroffene zum »bloßen Objekt im Staate« gemacht. Dies widerspricht der Menschenwürde. ${ }^{35}$

25 Brugger, JZ 2000, 165, 167.

26 Vgl. Brugger, aaO (Fn. 25), 169.

27 BGB1. II 1952, 685.

28 BGBl. II 1990, 246.

29 Von Münch/Kunig-Kunig, GG, 5. Auflage 2000, Art. 1, Rn. 22.

30 BVerfGE 30, 1, 25.

31 Von Münch/Kunig-Kunig, aaO (Fn. 29), Art. 1, Rn. 22; Sachs-Höfling, GG, 3. Auflage 2003, Art. 1, Rn. 12; Merten, JR 2003, 404, 406.

32 Eindringlich Sachs-Höfling, aaO (Fn. 31), Art. 1, Rn. 9; von Münch/Kunig-Kunig, aaO (Fn. 29), Art. 1, Rn. 6, 8.

33 Sachs-Höfling, aaO (Fn. 31), Art. 1, Rn. 60.

34 Von Münch/Kunig-Kunig, aaO (Fn. 29), Art. 1, Rn. 24; Sachs-Höfling, aaO (Fn. 31), Art. 1, Rn. 60; Haurand/Vahle, aaO (Fn. 4), 518 f.

35 Sogenannte Objektformel des BVerfG, vgl. BVerfGE 9, 89, 95; E 45, 187, 228; E 50, 166, 175; Sachs-Höfling, aaO (Fn. 31), Art. 1, Rn. 20; Hecker, aaO (Fn. 17), 215, dort Fn. 25; Merten, aaO (Fn. 31), 406. 
Stützen läßt sich dieses Ergebnis auch auf die Bestimmung des Art. 104 I 2 GG. ${ }^{36}$ Diese Norm verbietet die seelische oder körperliche Mißhandlung festgehaltener Personen. Insofern enthält die Regelung die Klarstellung eines sich bereits aus Art. 1 I GG ergebenden Verbotes. ${ }^{37}$ Unter »seelische Mißhandlung « fallen alle Maßnahmen, welche geeignet sind, die freie Willensbildung und das Erinnerungsvermögen zu beeinträchtigen. ${ }^{38}$ Darunter kann insbesondere die Androhung der Anwendung von Gewalt subsumiert werden. Der unmißverständliche Wortlaut von $\S 12$ IV HSOG ist Ausfluß dieser Grundsätze und Ausdruck einer bewußten gesetzgeberischen Entscheidung vor deren Hintergrund. Eine »Bewertungslücke« besteht aufgrund dessen nicht. Eine teleologische Reduktion von $\S 12$ IV HSOG und damit auch eine Androhung von Gewalt ist methodisch nicht zu rechtfertigen. ${ }^{39}$

Dem läßt sich nicht entgegnen, das Leben des Entführungsopfers bedürfe desselben Schutzes wie die Menschenwürde. ${ }^{40}$ Zweifellos erfahren auch das Leben und die körperliche Unversehrtheit Schutz durch das Grundgesetz (vgl. Art. 2 II 1 GG). Zutreffend ist, daß eine Grundrechtskollision vorliegt. Die Argumentation mit einer »Bewertungslücke« überschreitet aber verfassungsrechtliche Grenzen, indem sie die vom Grundgesetz selbst anerkannte besondere Wertigkeit der Menschenwürde unberücksichtigt läßt. Das BVerfG nennt die Menschenwürde den »höchsten Rechtswert «. ${ }^{41}$ Bereits durch den Wortlaut von Art. 1 I GG (»unantastbar «) hebt die Verfassung die Menschenwürde von den übrigen Grundrechten ab. Insofern kann eine Grundrechtskollision, jedenfalls wenn und soweit die Menschenwürde auf einer Seite im Streit steht, durch einen Rückgriff auf ihren »höheren Rang « bewältigt werden. ${ }^{42}$ Dies darf aber nicht als Abwägung mißverstanden werden. Eine solche findet gerade nicht statt. ${ }^{43}$ Brugger vermischt die grundrechtsdogmatisch zu unterscheidenden Kategorien des Schutzbereichs eines Grundrechts und der verfassungsrechtlichen Rechtfertigung eines Eingriffs in den Schutzbereich. Letztere Frage kann und darf hinsichtlich der Menschenwürde aufgrund ihrer Unantastbarkeit nicht aufgeworfen werden. Die Menschenwürde ist »abwägungsfest «. Jeder Eingriff in den Schutzbereich ist verfassungswidrig. ${ }^{44}$ Insofern handelt es sich hier nicht um eine Situation, in der sich einan-

36 Hecker, $\mathrm{aaO}$ (Fn. 17), 212 f.; Dombek, Akzente, BRAK Mitteilungen 2/2003 vom 15.04.2003; Düx, ZRP 2003, 180; vgl. LG Frankfurt am Main, StV 2003, 327.

37 Von Münch/Kunig-Kunig, GG, 3. Auflage 1996, Art. 104, Rn. 12; Merten, aaO (Fn. 31), 405.

38 Von Münch/Kunig-Kunig, aaO (Fn. 37), Art. 104, Rn. 15.

39 Jerouschek/Kölbel, aaO (Fn. 6), 617, insbesondere in Fn. 42.

40 So aber Brugger, aaO (Fn. 24), S. 8, dritte Spalte.

41 BVerfGE 45, 187, 227.

42 Von Münch/Kunig-von Münch, aaO (Fn. 29), Vorb. Art. 1-19, Rn. 46; von Münch/KunigKunig, aaO (Fn. 29), Art. 1, Rn. 4.

43 Sachs-Höfling, aaO (Fn. 31), Art. 1, Rn. 10, unter Hinweis auf BVerfGE 75, 369, 380; ebenso Merten, aaO (Fn. 31), 406 und Dreier-Dreier, Grundgesetz Kommentar, 2. Auflage 2004, Art. 1 I, Rn. 132. A.A.Jerouschek/Kölbel, aaO (Fn. 6), 618 f. und Maunz-Dürig-Herdegen, Kommentar zum Grundgesetz, Art. 1 Abs. 1, Rn. 45, der zwischen dem unantastbaren »Würdekern « und einem abwägungsoffenen, peripheren Schutzbereich« differenziert.

44 Von Münch/Kunig-Kunig, aaO (Fn. 29), Art. 1, Rn. 4; Merten, aaO (Fn. 31), 406. 
der widersprechende Bewertungen des Verfassungsgebers gegenüberstehen. Die Annahme einer »Bewertungslücke « geht fehl. Diese rechtliche Ausgangslage bemerkt schließlich auch Brugger. ${ }^{45}$ Daher versucht er, auf zwei verschiedenen Wegen die Annahme eines Wertungswiderspruches zu begründen:

Zum einen stuft Brugger das Recht auf Leben und körperliche Unversehrtheit des Entführungsopfers argumentativ auf die Ebene der Menschenwürde herauf. Das Leben sei Voraussetzung für die Menschenwürde und deshalb gleichermaßen wie diese geschützt. ${ }^{46}$ Bereits diese »Hochstufung « des Rechts auf Leben auf die Ebene der Menschenwürde ist nicht überzeugend. Letztlich wird dadurch die Entscheidung der Verfassung für die Menschenwürde als »höchstem Rechtswert ${ }^{47}$ konterkariert. Die herausgehobene Stellung der Menschenwürde wird beeinträchtigt. ${ }^{48}$ Die Menschenwürde soll aber gerade nach Art. 1 I GG höheren Schutz als das Recht auf Leben genießen. ${ }^{49}$

Im übrigen trägt der aus der argumentativen Aufwertung des Rechtsguts »Leben« gezogene Schluß, eine »Bewertungslücke« liege vor, nicht. Eine solche Lücke soll nach Brugger vorliegen, wenn »die Wertung (...) im Lichte anderer Normen, als unangemessen, ungerecht « erscheint. ${ }^{50}$ Der Begriff der Unangemessenheit erinnert an ein Element des Verhältnismäßigkeitsprinzips, namentlich die Zumutbarkeit bzw. Verhältnismäßigkeit im engeren Sinne. Nun darf aber die Kategorie der Verhältnismäßigkeit oder eine ihrer Unterarten im Rahmen von Art. 1 I GG keine Rolle spielen, soll nicht verkannt werden, daß Art. 1 I GG »unantastbar« und damit dem grundrechtlichen Abwägungsprozeß entzogen ist. ${ }^{51}$

Zudem ist zweifelhaft, ob das Verhältnismäßigkeitsprinzip überhaupt geeignet ist, die Kollision von zwei Rechtsgütern, die aus Bruggers Sicht beide in Art. 1 I GG wurzeln, zu bewältigen. Im ersten Sondervotum zum 2. Abtreibungsurteil führten die Richter Mahrenholz und Sommer aus, der Maßstab der Unzumutbarkeit werde der »Einzigartigkeit der Situation « nicht gerecht. ${ }^{52}$ Angesichts der Abhängigkeit der Beur-

$45 \mathrm{Vgl}$. den mit »Das absolute Verbot der Anwendung von Folter im deutschen und internationalen Recht « überschriebenen Gliederungspunkt II., Brugger, aaO (Fn. 25), 166. Diesen versieht Brugger - angesichts seiner Perspektive erstaunlich - nicht einmal mit einem Fragezeichen.

46 Brugger, aaO (Fn. 24), S. 8, dritte Spalte; ähnlich von Mangoldt/Klein/Starck-Starck, Bonner Grundgesetz, Bd. 1, 4. Auflage 1999, Art. 1 Abs. 1, Rn. 71. Diese argumentative »Hochstufung« übersehend Merten, aaO (Fn. 31), 407. Allgemein eine rechtfertigende Pflichtenkollision in den Fällen, in denen Würde gegen Würde steht, nicht ausschließen wollend Dreier-Dreier, aaO (Fn. 43), Art. 1 I, Rn. 133. Ebenso Wittreck, DÖV 2003, 873, 879-882.

47 BVerfGE 45, 187, 227.

48 Sachs-Höfling, aaO (Fn. 31), Art. 1, Rn. 11; Merten, aaO (Fn. 31), 407; Wittreck, aaO (Fn. 46), 878.

49 Von Münch/Kunig-Kunig, aaO (Fn. 29), Art. 1, Rn. 5; Wittreck, aaO (Fn. 46), 878. A.A. Maunz-Dürig-Herdegen, aaO (Fn. 43), Art. 1 Abs. 1, Rn. 45 (»normimmanente Konkretisierung des Würdeanspruches«); zu dieser Ansicht zu Recht kritisch Merten aaO (Fn. 31), 406.

50 Brugger, aaO (Fn. 25), 167.

51 Vgl. BVerfGE 75, 369, 380; Sachs-Höfling, aaO (Fn. 31), Art. 1, Rn. 10; Merten, aaO (Fn. 31), $406 \mathrm{f}$.

52 BVerfGE 88, 338, $345 \mathrm{ff}$. 
teilung der Konfliktlage von dem subjektiven Empfinden der jeweiligen Frau, verführten abstrakt-generelle Kriterien $\mathrm{zu} »$ Subsumierungen, deren Maßstäbe um so vager sind, je mehr die jeweilige Konfliktlage der Subjektivität der Frau verhaftet ist $« .{ }^{53}$ In dem uns interessierenden Fall sollen sich nach Brugger zwei »höchste Rechtswerte« unterschiedlicher Individuen gegenüberstehen. In dieser Ausnahmesituation können Abwägungsprozesse der handelnden staatlichen Stellen ebenfalls normativ kaum gesteuert werden ${ }^{54}$ Der dafür zu zahlende $»$ Preis « besteht in einem rechtlich nicht einzuhegenden »Freiraum « für Gefahrenabwehr- und Polizeibehörden, welche über die Androhung bzw. Anwendung von Folter zu entscheiden haben. Auch aus der Perspektive der handelnden Träger staatlicher Gewalt ist das Übermaßverbot untauglich: Es muß in der praktischen Anwendung versagen. Denn die Vermeidung des Übermaßes setzt die Kenntnis des Maßes voraus. Eine Grenzziehung zwischen Foltern und (legalem) Noch-nicht-Foltern ist aber praktisch unmöglich. ${ }^{55}$ Daher muß jeder Versuch scheitern, das rechte Maß im konkreten Einzelfall festzulegen. Den Polizeibeamten ist es deshalb nicht zuzumuten, über die Androhung von Gewalt oder gar Anwendung von Gewalt zur Erlangung von Informationen entscheiden zu müssen. ${ }^{56}$

Abgesehen davon, führt die Ansicht Bruggers zu (aus seiner Sicht vermutlich unerwünschten) Konsequenzen für die Frage der Verfassungsmäßigkeit des »finalen Rettungsschusses«. Träfe es zu, daß das Rechtsgut »Leben« verfassungsrechtlich auf gleicher Ebene wie die Menschenwürde steht, weil jenes diese bedinge, könnte der »finale Rettungsschuß « einen Eingriff in die Menschenwürde des Betroffenen darstellen. Ein Eingriff in das Schutzgut »Leben« würde dann zwingend eine Verletzung der Menschenwürde indizieren. ${ }^{57}$ Die entsprechenden einfachgesetzlichen Ermächtigungsgrundlagen, die einen derartigen Eingriff gestatten, könnten als verfassungswidrig angesehen werden.

Um die Rechtmäßigkeit der Androhung von Gewalt zu belegen, wird zum anderen die Pflicht des Staates ins Feld geführt, das Entführungsopfer vor Eingriffen in seine Grundrechte zu schützen. ${ }^{58}$ Die Menschenwürde des Entführungsopfers sei unmittelbar tangiert. Das Opfer werde durch seinen Entführer zum »bloßen Objekt« herabgewürdigt, weil dieser Leib und Leben des Opfers zur Erpressung instrumentalisiere. ${ }^{59}$

Grundrechte können - über ihre traditionelle Abwehrfunktion gegen den Staat hinausgehend - »als Elemente objektiver Ordnung ${ }^{60}$ auch staatliche Schutzpflichten begrün-

53 Sondervotum Mahrenholz und Sommer, BVerfGE 88, 338, 346.

54 Siehe dazu näher C.II.1.c). Dies ist Wittreck, aaO (Fn. 46), 881 f., entgegenzuhalten, nach dessen Ansicht Opfer- und Täterwürde im konkreten Einzelfall abzuwägen seien.

55 Grasnick, aaO (Fn. 6), S. 43, fünfte und sechste Spalte; Jerouschek/Kölbel, aaO (Fn. 6), 618; skeptisch auch Miehe, aaO (Fn. 5), 1220, ohne daraus aber Konsequenzen zu ziehen. A.A. Schreiber, aaO (Fn. 6), XVI.

56 Dies gilt insbesondere im Hinblick auf die Grundrechtsbelange des mit der Folter betrauten Trägers hoheitlicher Gewalt, vgl. Jerouschek/Kölbel, aaO (Fn. 6), 618.

57 Vgl. Sachs-Höfling, aaO (Fn. 31), Art. 1, Rn. 11.

58 Brugger, aaO (Fn. 24), S. 8, dritte Spalte; vgl. früher schon Brugger, aaO (Fn. 25), $170 \mathrm{f.}$

59 Brugger, aaO (Fn. 24), S. 8, dritte Spalte.

60 Kritisch gegenüber dieser Begründung aber von Münch/Kunig-von Münch, aaO (Fn. 29), Vorb. Art. 1-19, Rn. 22 a.E. 
den. ${ }^{61} \mathrm{Zu}$ Recht macht von Münch aber darauf aufmerksam, der so gewährte Schutz könne häufig ausschließlich durch Eingriffe in Grundrechte anderer verwirklicht werden. ${ }^{62}$ Dies ist nur dann nicht von vornherein zu beanstanden, wenn Grundrechte betroffen sind, in welche von Verfassungs wegen eingegriffen werden darf. Das ist bei der Menschenwürde indes nicht der Fall. Denn das Grundgesetz bezeichnet diese - im Unterschied zum Recht auf Leben und körperliche Unversehrtheit (Art. 2 II 1 GG) - als »unantastbar «. Deshalb erklärt Art. 79 III GG jede Änderung des Grundgesetzes, durch welche Grundsätze des Art. 1 GG »berührt« werden, für unzulässig (sogenannte »Ewigkeitsklausel«). Dies darf nicht durch den Rekurs auf staatliche Schutzpflichten überspielt werden, um zum Schluß zu gelangen, »der rechtstreue Bürger verdient den Vorrang $\ll{ }^{63}$ Diese Folgerung ist Ergebnis einer Abwägung, die im Rahmen von Art. 1 I GG systemwidrig und unzulässig ist. Die Menschenwürde darf gerade nicht »angetastet« werden. So hat denn auch das BVerfG staatliche Schutzpflichten nur für Fälle bejaht, in denen es um den Schutz von Leib und Leben ging, ohne daß damit zugleich ein Einbruch in die »unantastbare « Menschenwürde verbunden war. ${ }^{64}$

Die völkerrechtlichen Regelungen unterstreichen das bisher gefundene Ergebnis. Insbesondere sind Inhalt und Entwicklungsstand der EMRK, welche nach herrschender Auffassung über Art. 59 II GG den Rang eines einfachen Bundesgesetzes hat, ${ }^{65}$ bei der Auslegung der Verfassung zu berücksichtigen. ${ }^{66}$

Nach Art. 3 EMRK darf »niemand der Folter (...) unterworfen werden«. Folter ist nach § 1 I der Anti-Folterkonvention der Vereinten Nationen, welche Art. 3 EMRK insofern ergänzt, ${ }^{67} »$ jede Handlung, durch die einer Person vorsätzlich große körperliche oder seelische Schmerzen oder Leiden zugefügt werden, zum Beispiel um von ihr oder einem Dritten eine Aussage oder ein Geständnis zu erlangen ..., wenn diese Schmerzen oder Leiden durch einen Angehörigen des öffentlichen Dienstes oder eine andere in amtlicher Eigenschaft handelnde Person, auf deren Veranlassung oder mit deren ausdrücklichem oder stillschweigendem Einverständnis verursacht werden.« Diese Definition kann zur Auslegung von Art. 3 EMRK herangezogen werden. ${ }^{68}$ Art. 3 EMRK erfaßt auch die Androhung von Gewalt zur Erzwingung einer Information. Denn diese kann unter das Merkmal »Zufügung großer seelischer Schmerzen« subsumiert werden. ${ }^{69}$

61 BVerfGE 46, 160; E 88, 203, 251.

62 Von Münch/Kunig-von Münch, aaO (Fn. 29), Vorb. Art. 1-19, Rn. 22 a.E.

63 So aber Brugger, aaO (Fn. 24), S. 8, dritte Spalte. Wie hier Schreiber, aaO (Fn. 6), XIV; Hecker, aaO (Fn. 17), 213 f.; Merten, aaO (Fn. 31), 407 f.

64 Vgl. exemplarisch BVerfGE 46, 160; E 88, 203, 251, jeweils m.w.N.

65 Von Münch/Kunig-Rojahn, GG, 5. Auflage 2001, Art. 59, Rn. 38 m.w.N.

66 Von Münch/Kunig-Rojahn, aaO (Fn. 65), Art. 59, Rn. 38 e.

67 Löwe-Rosenberg-Gollwitzer, StPO, 24. Auflage 1996, Art. 3 EMRK, Rn. 4.

68 EGMR NJW 2001, 56, 59 f.; Meyer-Ladewig, EMRK, 2003, Art. 3, Rn. 7; Löwe-Rosenberg-Gollwitzer, aaO (Fn. 67), Art. 3 EMRK, Rn. 19; Frowein/Peukert, EMRK, 2. Auflage 1996, Art. 3, Rn. 6.

69 EGMR NJW 1990, 2183; Frowein/Peukert, aaO (Fn. 68), Art. 3, Rn. 5, 17; Löwe-Rosenberg-Gollwitzer, aaO (Fn. 67), Art. 3 EMRK, Rn. 18; Schroeder, ZRP 2003, 180; ähnlich Hecker, aaO (Fn. 17), 217; so auch i.E. LG Frankfurt am Main, StV 2003, 327 und der Geschäftsführende Ausschuß der Arbeitsgemeinschaft Strafrecht und Strafrechtsausschuß des DAV, Dokumentation, StV 2003, 255. 
Daraus, daß Art. 2 II EMRK unter bestimmten Voraussetzungen Tötungen zuläßt, ergibt sich entgegen der Auffassung Bruggers ${ }^{70}$ nichts anderes. Dieser vertritt die Ansicht, Art. 3 EMRK sei in Konkordanz mit Art. 2 II a EMRK zu bringen. Art. 2 II a EMRK bestimmt, daß eine Tötung zulässig ist, wenn sie sich »aus einer unbedingt erforderlichen Gewaltanwendung ergibt, um die Verteidigung eines Menschen gegenüber rechtswidriger Gewaltanwendung sicherzustellen«. In »unvermeidbaren Konfliktfällen « wie hier sei der Anwendungsbereich von Art. 3 EMRK teleologisch zu reduzieren. ${ }^{71}$ Gegen eine derartige teleologische Reduktion von Art. 3 EMRK ist einzuwenden, daß Art. 3 EMRK lex specialis gegenüber Art. 2 II a EMRK ist. Der Unterschied beider Normen liegt darin, daß Art. 3 EMRK dem Schutz der Menschenwürde dient, wohingegen Art. 2 II a EMRK Eingriffe in das Recht auf Leben unter bestimmten Voraussetzungen rechtfertigt. Daher greift es zu kurz, Art. 3 EMRK nur in bezug auf das Mittel für spezifischer als Art. 2 II a EMRK zu erachten. ${ }^{72}$ Der Grund der Unterscheidung beider Regelungen liegt in dem Rangverhältnis der betroffenen Rechtsgüter - Leben auf der einen, Menschenwürde auf der anderen Seite. Das Verbot des Art. 3 EMRK ist notstandsfest garantiert. Nicht einmal im Fall eines Krieges oder eines sonstigen öffentlichen Notstandes darf das Folterverbot von den Vertragsparteien außer Kraft gesetzt werden (vgl. die ausdrückliche Regelung in Art. 15 II EMRK). ${ }^{73}$ In gleicher Weise bestimmt Art. 2 II der Antifolter-Konvention, daß außergewöhnliche Umstände gleich welcher Art nicht als Rechtfertigungsgründe für Folter geltend gemacht werden dürfen. ${ }^{74}$ Daher fehlt es auch hier an einer Lücke, welche aber Voraussetzung für eine teleologische Reduktion ist. ${ }^{75}$

b) Strafrechtliche Rechtfertigung des Ermittlungsbeamten?

Aber auch das Strafrecht bietet keinen Rechtfertigungsgrund. Umstritten ist schon, ob sich Träger staatlicher Gewalt überhaupt auf Rechtfertigungsgründe des StGB berufen dürfen. ${ }^{76}$

Zutreffend wird darauf hingewiesen, daß die Beantwortung dieser Frage davon abhängt, ob die strafrechtlichen Rechtfertigungsgründe im Einzelfall durch besondere öffentlich-rechtliche Regelungen für den Bereich hoheitlichen Handelns begrenzt werden. ${ }^{77}$ Vorliegend sind die einfachgesetzlichen Bestimmungen der $§ \S 32,34$ StGB im

70 Brugger, aaO (Fn. 24), S. 8, vierte Spalte; Brugger, aaO (Fn. 25), 170.

71 Brugger, aaO (Fn. 24), S. 8, vierte Spalte; vgl. Brugger, aaO (Fn. 25), 170.

72 A.A. Brugger, aaO (Fn. 25), 170, der Art. 2 II a EMRK lediglich im Hinblick auf »Agressionsakte Dritter « für spezieller hält.

73 Merten, aaO (Fn. 31), 405; Geschäftsführender Ausschuß der Arbeitsgemeinschaft Strafrecht und Strafrechtsausschuß des DAV, aaO (Fn. 69), 255.

74 Vgl. Sachs-Höfling, aaO (Fn. 31), Art. 1, in Fn. 69; Düx, ZRP 2003, 180; insoweit zutreffend Maunz-Dürig-Herdegen, aaO (Fn. 43), Art. 1 Abs. 1, Rn. 90.

75 Um Wiederholungen zu vermeiden, sei auf die obige, parallele Argumentation zum deutschen Verfassungsrecht verwiesen.

76 Zum Streitstand Beaucamp, JA 2003, 402; Leipziger Kommentar-Hirsch, aaO (Fn. 11), Vor $\S 32$, Rn. 153; § 34, Rn. 7 ff., 18, jeweils m.w.N. Offenlassend Kubink, NJW 2003, Heft 24, XII, XIV.

77 Schönke/Schröder-Lenckner/Perron, StGB, 26. Auflage 2001, § 32, Rn. 42 b. 
Lichte von Art. 1 I GG zu sehen. Denn die staatlich veranlaßte Androhung von Gewalt, um eine Information zu erlangen, beeinträchtigt die Menschenwürde des Befragten. Nach Art. 1 I GG ist die Menschenwürde aber »unantastbar«. Daher darf sich ein Träger hoheitlicher Gewalt nicht auf strafrechtliche Rechtfertigungsgründe wie $\S \S 32,34$ StGB berufen, wenn und soweit wie hier der Schutzbereich von Art. 1 I GG tangiert ist. ${ }^{78} \mathrm{Da} \S 54$ II HSOG davon ausgeht, auch Amtsträger können sich auf strafrechtliche Rechtfertigungsgründe berufen (»Die zivil- und strafrechtlichen Wirkungen über Notwehr und Notstand bleiben unberührt«), steht dazu nicht im Widerspruch. ${ }^{79}$ Denn Bundesrecht geht nach der Kompetenzordnung des Grundgesetzes entgegenstehendem Landesrecht vor (Art. $31 \mathrm{GG}$ ). Auch daraus, daß die herrschende Lehre im Strafrecht beim Schußwaffengebrauch eine strafrechtliche Rechtfertigung staatlicher Hoheitsträger nach $\S \S 32,34$ StGB zuläßt, ergibt sich nichts anderes. ${ }^{80} \mathrm{Im}$ Unterschied zum »Fall Daschner« findet beim polizeilichen Schußwaffengebrauch ein Eingriff in Art. 2 II 1 GG statt. Dieses Grundrecht ist nicht schrankenlos gewährleistet, sondern enthält in Art. 2 II 3 GG einen allgemeinen Gesetzesvorbehalt. Eingriffe sind also möglich, sofern sie auf einer (verfassungsgemäßen) Ermächtigungsgrundlage beruhen.

Abgesehen davon, lassen sich auch die völkerrechtlichen Bestimmungen, welche Folter ausnahmslos ${ }^{81}$ ausschließen, für die Unanwendbarkeit von $\S \S 32,34$ StGB hinsichtlich der Erzwingung der Auskunftspflicht anführen. ${ }^{82}$ Denn diese gehen den strafrechtlichen Rechtfertigungsgründen als leges speciales sowie leges posteriores vor. ${ }^{83}$

c) Exkurs: Rechtspolitische Einwände gegen die Absolutheit des Folterverbotes Rechtspolitisch werden verschiedene Einwände gegen die Absolutheit des Folterverbots erhoben. Es wird auf die Aufgabe des Staates hingewiesen, kraft seines Gewaltmonopols Zwang effektiv zur Rechtswahrung anzuwenden, da ihm die Menschen ihren Schutz überantwortet hätten. ${ }^{84}$ Komme der Staat dieser Aufgabe nicht nach, wendeten sich die Menschen enttäuscht von ihm ab und kehrten zum Faustrecht zurück. Aufgrund dessen müsse der »Vorrang der Wahrung von Zivilität« in Ausnahmefällen aufgegeben werden. ${ }^{85}$ An dieser Stelle wird die Effektivität der Gefahrenabwehr bzw. Strafverfolgung gegen das Rechtsstaatsprinzip, Art. 20 III GG, ausgespielt. Sofern es überhaupt zutreffend ist, daß sich die »Menschen« enttäuscht vom Staat ab-

78 Gegen eine derartige Anknüpfung an Verfassungsrecht Jerouschek/Kölbel, aaO (Fn. 6), 619 f. Noch weitgehender meint Wittreck, aaO (Fn. 46), 877, unter Berücksichtigung der Menschenwürde des Entführungsopfers sei die Tat gem. § 32 I StGB gerechtfertigt.

79 Daran anknüpfend aber Hecker, aaO (Fn. 17), 215, insbesondere Fn. 27.

80 Vgl. dazu Schönke/Schröder-Lenckner/Perron, aaO (Fn. 77), § 32, Rn. 42 c m.w.N.

81 A.A. Jerouschek/Kölbel, aaO (Fn. 6), 620, insbesondere in Fn 74.

82 A.A. Schreiber, aaO (Fn. 6), XVI, der meint, bei einer gerechtfertigten Gewaltandrohung liege begrifflich keine Folter vor. Noch weiter scheint Maunz-Dürig-Herdegen, aaO (Fn. 43), Art. 1 Abs. 1, Rn. 47, gehen zu wollen, wenn er einen Ausschluß der Unrechtsfolgen im Strafrecht »von Verfassungs wegen« für möglich hält.

83 Insofern zutreffend Brugger, aaO (Fn. 25), 168.

84 Brugger, aaO (Fn. 24), S. 8, vierte Spalte.

85 Brugger, aaO (Fn. 24), S. 8, fünfte Spalte. 
und dem Faustrecht zuwendeten, bleibt doch zweifelhaft, ob die angesonnene Abhilfe als ausreichend erachtet werden würde. Es steht zu befürchten, daß eine Ausnahme vom Folterverbot schon bald - selbstverständlich im Interesse der »Effektivität der Rechtswahrung « - für nicht genügend gehalten und in praxi eine erhebliche Ausweitung der Ausnahme vom Folterverbot vorgenommen werden würde. Die Gefahr eines »Dammbruchs « ist nicht von der Hand zu weisen. ${ }^{86}$ Begreift man den Rechtsstaat und die Herrschaft des Rechts als Mittel zur »Zähmung « staatlicher Gewalt, muß die Schaffung von Ausnahmen von ausdrücklichen, unmißverständlichen Verboten für staatliches Handeln bedenklich stimmen. Dies gilt umso mehr, als auch der von Brugger de lege ferenda vorgeschlagene Tatbestand ${ }^{87}$ nicht vor expansiver Anwendung gefeit ist. Eine ex-post-Kontrolle wird nicht genügen. ${ }^{88}$ Denn im Alltag werden nicht Juristen mit der Auslegung des Tatbestandes befaßt sein, sondern Gefahrenabwehrund Polizeibehörden sehen sich vor die Aufgabe gestellt, unter Zeitdruck über das Vorliegen des Tatbestandes zu befinden. »Im Interesse einer effektiven Rechtswahrung « wird dann tendenziell die Zulässigkeit von Folter bejaht werden, ohne daß ein Jurist hinzugezogen wurde. Man könnte dem insofern abhelfen wollen, als man einen sogenannten Richtervorbehalt in den Tatbestand der Ausnahme vom Folterverbot einführt. Dann bedürfte die Anwendung von Folter der Zustimmung eines Richters. Viele Vorschriften, welche einen Richtervorbehalt enthalten, sehen aber zugleich vor, daß bei »Gefahr im Verzug « die Zustimmung eines Richters entbehrlich ist. ${ }^{89}$ Das BVerfG machte kürzlich darauf aufmerksam, daß Strafverfolgungsbehörden ihrer geringen Neigung, der gesetzlichen Verpflichtung gemäß Richter einzuschalten, sich des öfteren entledigen, indem sie ohne nähere Begründung »Gefahr im Verzug « bejahen. ${ }^{90}$ Dieses Verhalten läßt befürchten, daß es in dem hier interessierenden Zusammenhang nicht anders wäre. ${ }^{91}$ Deshalb und wegen der verfassungsrechtlichen Bedeutung der Menschenwürde wäre es rechtsstaatlich geboten, ausnahmslos die Einschaltung eines Richters vorzuschreiben. Andererseits kommt darin, daß bei »Gefahr im Verzug « kein Richter der Maßnahme zustimmen muß, das Interesse an effektiver Rechtsverfolgung zum Ausdruck. Wenn aber bereits der Vorschlag einer Ausnahmevorschrift vom Folterverbot tatbestandsmäßig das Vorliegen einer »klaren, unmittelbaren, erheblichen Gefahr $" ~ " 2$ vorsehen soll, dann liegt es nahe, die Einschaltung eines Richters gar nicht erst zur Voraussetzung für die Rechtmäßigkeit der Anwendung von Folter zu machen, da doch »Gefahr im Verzug « gewissermaßen Tatbestandsmerkmal ist. Bejaht man also die Notwendigkeit einer Ausnahme vom Folterverbot für den Fall einer »klaren, unmittelbaren, erheblichen Gefahr« mit der Begründung, dies sei im Interesse »effek-

86 Jerouschek/Kölbel, aaO (Fn. 6), 619.

87 Vgl. Brugger, aaO (Fn. 24), S. 8, fünfte Spalte.

88 A.A. Brugger, aaO (Fn. 24), S. 8, fünfte Spalte: »Diese Merkmale sind Juristen vertraut, sie sind justitiabel und bestimmt $(\ldots) \ll$.

89 Vgl. zum Beispiel 100 I, 100 b I, 100 d I, 105 I StPO.

90 BVerfG NStZ 2001, 382 = NJW 2001, 1121, 1122.

91 Vgl. Helmken, StV 2003, 193, passim, zum Leerlaufen des Richtervorbehalts in der strafprozessualen Praxis.

92 Vgl. Brugger, aaO (Fn. 24), S. 8, fünfte Spalte. 
tiver Rechtsbewahrung « geboten, muß man - konsequenterweise - auch auf die Einführung eines Richtervorbehalts verzichten. ${ }^{93}$ Auf die nachträgliche Kontrolle durch »Juristen « zu setzen, ist aber nicht mehr als ein schwacher Trost und befriedigt angesichts der Bedeutung der Menschenwürde nicht. Jene Kontrolle ist zudem ungeeignet, einer uferlosen Anwendung der vorgeschlagenen Ausnahme vom Folterverbot das Wasser abzugraben.

d) Verwerflichkeit des Verhaltens

Da aus rechtlichen Gründen ${ }^{94}$ keine Rechtfertigungsgründe Platz greifen, bleibt noch die Frage nach der Verwerflichkeit des Verhaltens (§ 240 II StGB).

Verwerflichkeit eines Verhaltens liegt vor, wenn der Zusammenhang von Zweck und Mittel in erhöhtem Maß als sozialethisch mißbilligenswert anzusehen ist. ${ }^{95}$ »Zweck « ist das Verhalten, welches der Täter vom Opfer erzwungen hat oder erzwingen will. ${ }^{96}$ Die Androhung von Gewalt durch den Polizeibeamten diente dem Ziel, den Aufenthaltsort des Entführungsopfers zu erfahren. Verwerflichkeit ist nicht bereits deshalb gegeben, weil die Verwirklichung der Drohung rechtswidrig wäre. ${ }^{97}$ Vielmehr kann es die Verwerflichkeit indizieren, wenn das Nötigungsmittel als solches gegen die Rechtsordnung verstößt. ${ }^{98}$ Das ist hier der Fall, da nicht erst die Anwendung von Gewalt zur Erlangung einer Äußerung, sondern schon deren Androhung rechtswidrig war. ${ }^{99}$ Dies gilt auch unter Berücksichtigung des Verhältnisses zum Nötigungszweck. Die Tatsache, daß Magnus G. polizeirechtlich gem. § 12 II 1 HSOG zur Auskunft verpflichtet war, schließt die Nötigung nicht aus. Denn wie schon private Gläubiger nicht davon entbunden sind, sich zur Durchsetzung ihrer zivilrechtlichen Ansprüche der vom Recht zur Verfügung gestellten Mittel zu bedienen, ${ }^{100}$ muß dies erst recht für staatliche Hoheitsträger hinsichtlich der Durchsetzung öffentlich-rechtlicher Pflichten gegenüber dem Bürger gelten. Hier stehen aber die Vorschriften des HSOG einer Durchsetzung der Auskunftspflicht durch Androhung von Gewalt klar entgegen.

2. Strafrechtliche Legitimation des Verhaltens von Daschner

Daschner kann sich ebensowenig wie der ermittelnde Polizeibeamte auf Rechtfertigungsgründe berufen. ${ }^{101}$

Seiner strafrechtlichen Verantwortlichkeit steht nicht entgegen, daß ihm die nach $\S 17$ StGB erforderliche Einsicht fehlte, Unrecht zu tun. Daschner befand sich viel-

93 Folgerichtig verzichtet Brugger auf das Erfordernis einer Zustimmung des Richters, aaO (Fn. 24), S. 8, fünfte Spalte.

94 Hingegen nimmt Schreiber, aaO (Fn. 6), XVI, einen Erlaubnistatbestandsirrtum an, da das Entführungsopfer im Zeitpunkt der Gewaltandrohung bereits tot war. So wohl auch Jerouschek/Kölbel, aaO (Fn. 6), 620, dort Fn. 75.

95 Schönke/Schröder-Eser, aaO (Fn. 77), § 240, Rn. 17.

96 Tröndle/Fischer, aaO (Fn. 5), § 240, Rn. 43.

97 Tröndle/Fischer, aaO (Fn. 5), § 240, Rn. 51.

98 Schönke/Schröder-Eser, aaO (Fn. 77), § 240, Rn. 19.

99 Siehe C.II.1.a) und C.II.1.b).

100 Vgl. Schönke/Schröder-Eser, aaO (Fn. 77), § 240, Rn. 21.

101 Vgl. C.II.1.a) und C.II.1.b). 
mehr in einem vermeidbaren Verbotsirrtum. Der Täter handelt ohne Unrechtsbewußtsein, wenn er die Grenzen eines anerkannten Rechtfertigungsgrundes überschreitet und sich bei Begehung der Tat in einem Irrtum über dessen rechtliche Voraussetzungen befand. ${ }^{102}$ Gegen einen Irrtum Daschners über die rechtlichen Voraussetzungen eines Rechtfertigungsgrundes spricht nicht, daß er laut dem von ihm gefertigten Aktenvermerk von Anfang an davon ausging, sein Verhalten werde »juristisch überprüft « werden. Zwar könnte er damit zum Ausdruck gebracht haben, er sei sich der Rechtswidrigkeit seines Verhaltens bewußt gewesen. Diese Schlußfolgerung wäre aber voreilig. Denn nach seinem Aktenvermerk nahm Daschner eine Abwägung des Rechts auf Leben des Entführungsopfers und des Recht auf körperliche Unversehrtheit des Entführers vor. Aufgrund dessen gelangte er zum Schluß, er dürfe den Polizeibeamten anweisen, Magnus G. die Anwendung von Gewalt in Aussicht zu stellen. Bei der Subsumtion unter $\S \S 12$ I 1, 52 I HSOG verkannte Daschner, daß die Androhung von Gewalt einen Eingriff in die Menschenwürde des Entführers darstellte. Eine Abwägung durfte Daschner in diesem Zusammenhang nicht vornehmen, da die Menschenwürde »unantastbar « und damit abwägungsfest ist. ${ }^{103}$ Des weiteren verbieten $\S 12$ IV HSOG sowie $\S 52$ II HSOG jede Androhung von Gewalt zur Erlangung einer Information. Indem er glaubte, in diesem Rahmen Rechtsgüter abwägen zu dürfen, als Ergebnis dieser Abwägung ein Überwiegen des Rechts auf Leben des Entführungsopfers konstatierte und daraufhin die Anweisung zur Androhung von Gewalt gab, irrte Daschner über Voraussetzungen polizeirechtlicher Ermächtigungsgrundlagen.

Dieser Irrtum war jedoch vermeidbar. ${ }^{104}$ Vermeidbar ist ein Verbotsirrtum, wenn dem Täter sein Vorhaben unter Berücksichtigung seiner Fähigkeiten und Kenntnisse hätte Anlaß geben müssen, über dessen mögliche Rechtswidrigkeit nachzudenken oder sich zu erkundigen, und er auf diesem Wege zur Unrechtseinsicht gekommen wäre. ${ }^{105}$ Besonders strenge Anforderungen sind an einen Rechtskundigen zu stellen. Von diesem kann angenommen werden, daß er die Tragweite gesetzlicher Vorschriften auf Grund seiner Berufsausbildung zu erkennen vermag. ${ }^{106}$ Angesichts seiner Position als Vizepräsident der Frankfurter Polizeibehörde kann davon ausgegangen werden, daß Daschner mit den einschlägigen rechtlichen Vorschriften vertraut sein mußte. Insbesondere ergab sich die Rechtswidrigkeit seiner Anweisung unmittelbar aus dem eindeutigen und klaren Wortlaut des $§ 12$ IV HSOG bzw. $§ 52$ II HSOG. Wie der Umstand, daß er einen Aktenvermerk über sein Vorgehen anfertigte, zeigt, hielt Daschner es für möglich, sein Verhalten könnte rechtlich anzuzweifeln sein.

Auch kann sich Daschner nicht auf einen entschuldigenden Notstand gem. $§ 35$ StGB berufen.

102 Vgl. Tröndle/Fischer, aaO (Fn. 5), § 17, Rn. 11; § 32, Rn. 27 a.E.

103 Siehe C.II.1.a).

104 Vgl. auch Grasnick, aaO (Fn. 6), S. 43, sechste Spalte. A.A. wohl Kubink, aaO (Fn. 76), XIV.

105 Tröndle/Fischer, aaO (Fn. 5), § 17, Rn. 7.

106 OLG Köln NJW 1996, 473; Tröndle/Fischer, aaO (Fn. 5), § 17, Rn. 8; Wittreck, aaO (Fn. 46), 877. 
Denn dies setzt eine Gefahr für Leben, Leib oder Freiheit einer anderen, dem Täter nahestehenden Person voraus. Ein solches Näheverhältnis besteht, wenn ein Mensch, dem Täter so verbunden ist, daß dieser eine Gefahr für jenen auch für sich selbst als Drucksituation empfindet. Dies wird bejaht für Verwandte, die nicht Angehörige im Sinne von $\S 11$ I Nr. 1 a) StGB sind, enge Freunde oder auch langjährige Bedienstete. ${ }^{107}$ Demnach ist Jakob von Metzler im Verhältnis zu Daschner nicht als andere nahestehende Person im Sinne von $§ 35$ StGB zu qualifizieren.

\section{Besonders schwerer Fall?}

Die Staatsanwaltschaft geht von einem besonders schweren Fall der Nötigung aus ( $\$ 240$ IV 2 Nr. 3 StGB). Dies setzt voraus, daß der Täter seine Befugnisse als Amtsträger mißbrauchte. Daschner ist zwar Amtsträger gem. § 11 I Nr. 2 StGB. Da die Amtsträgereigenschaft besonderes persönliches Merkmal ist, auf das §28 II StGB entsprechende Anwendung findet, kann auch der seine Befugnisse als Amtsträger mißbrauchende Teilnehmer das Regelbeispiel verwirklichen. ${ }^{108}$ Daher steht einer Anwendung von $\S 240$ IV 2 Nr. 3 StGB nicht bereits entgegen, daß Daschner Anstifter ist.

Mißbrauch der Befugnisse als Amtsträger heißt jedoch, daß der Täter Zwangsmittel, für deren Anwendung er grundsätzlich zuständig ist, mit verwerflicher Zweck-MittelRelation einsetzt. ${ }^{109}$ Daschner ist als Polizeibeamter - abstrakt gesehen - zur Anwendung unmittelbaren Zwanges befugt. Angesichts der hohen Strafdrohung und unter Berücksichtigung der übrigen Regelbeispiele muß die Strafzumessungsregel jedoch auf grobe Mißbrauchshandlungen beschränkt werden. ${ }^{110} \mathrm{Zu}$ berücksichtigen ist an dieser Stelle, daß die Drohung nicht in die Tat umgesetzt wurde. Zudem handelte es sich um eine Ausnahmesituation. ${ }^{111}$ Daraus kann man schließen, eine grobe Mißbrauchshandlung liege nicht vor.

\section{Ausblick}

Sowohl das hier gefundene Ergebnis, Daschner habe sich gem. §§ 240 I, 26 StGB strafbar gemacht, als auch die dem weitgehend entsprechende ${ }^{112}$ Entscheidung der Staatsanwaltschaft Frankfurt am Main, Anklage gegen ihn zu erheben, mögen in der breiten Öffentlichkeit vor dem Hintergrund des Todes des Jakob von Metzler auf Unverständnis stoßen. Gleichwohl sollte der »Fall Daschner« nicht als Vehikel dafür genutzt werden, die staatlich veranlaßte Androhung von Gewalt zur Erlangung von Informationen de lege ferenda zu legitimieren. Zum einen stünde die Verfassungskon-

107 Tröndle/Fischer, aaO (Fn. 5), § 35, Rn. 7.

108 Leipziger Kommentar-Träger/Altvater, StGB, 11. Auflage 2002, § 240, Rn. 123.

109 Tröndle/Fischer, aaO (Fn. 5), § 240, Rn. 61.

110 Leipziger Kommentar-Träger/Altvater, aaO (Fn. 108), § 240, Rn. 123; a.A. (weitergehend) Schönke/Schröder-Eser, aaO (Fn. 77), § 240, Rn. 38: Wenn der Täter »gesetz- oder pflichtwidrig « von seinen Befugnissen Gebrauch macht.

111 Haurand/Vahle, aaO (Fn. 4), 520.

112 Der einzige Unterschied im Ergebnis besteht darin, daß die Staatsanwaltschaft Frankfurt am Main von einer Anstiftung zur Nötigung in einem besonders schweren Fall ausgeht. 
formität einer solchen polizeirechtlichen Ermächtigungsgrundlage in Anbetracht von Art. 1 I GG sehr in Frage. Zum anderen wäre damit ein Einbruch in die rechtsstaatlich geschützte Sphäre des Einzelnen verbunden, der sich auch nicht durch den Gesichtspunkt der Effektivität der Gefahrenabwehr legitimieren ließe. Wohl begründete Grenzen staatlicher Gewalt dürfen nicht abgebaut werden, um nicht Gefahr zu laufen, daß solche, (zunächst) für Ausnahmefälle gedachten Normen eine derartige Ausweitung erfahren, daß sie im Laufe der Zeit nicht mehr als Ausnahme begriffen werden. Gerade in der Unnachgiebigkeit gegenüber einer vox populi, welche sich für eine Folterung von Beschuldigten in Ausnahmefällen ausspricht, erweist sich die Qualität des Rechtsstaates. $^{113}$

113 Grasnick, aaO (Fn. 6), S. 43, vierte und fünfte Spalte, meint sogar, ein Nachgeben in dieser Frage bedeute das Ende des Rechtsstaates. 